\title{
Is there a Role for Stereotactic Biopsy of Unresectable High-Grade Gliomas? A Retrospective Cohort Study of Short-Term Morbidity and Mortality
}

ISSN: 2637-7748

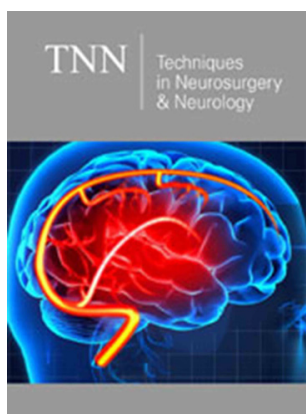

*Corresponding author: David Peters, Carolinas Medical Center, USA

Submission:

Published: 侮February 10, 2021

Volume 4 - Issue 1

How to cite this article: David R Peters, Anthony LA, James TS, Renee Kelly, Margaret Boltes, et al. Is there a Role for Stereotactic Biopsy of Unresectable High-Grade Gliomas? A Retrospective Cohort Study of Short-Term Morbidity and Mortality. Tech Neurosurg Neurol. 4(1). TNN. 000578. 2021.

DOI: 10.31031/TNN.2021.04.000578

Copyright@ David Peters, This article is distributed under the terms of the Creative Commons Attribution 4.0 International License, which permits unrestricted use and redistribution provided that the original author and source are credited.
David R Peters ${ }^{1,2 *}$, Anthony L Asher ${ }^{1,2}$, James T Symanowski ${ }^{3}$, Renee Kelly ${ }^{2}$, Margaret Boltes $^{2}$, Scott D Wait ${ }^{1,2}$, Mark D Smith ${ }^{1,2}$, Sarah C Jernigan ${ }^{1,2}$, Daniel Haggstrom $^{3}$, Ashley Sumrall ${ }^{3}$, Roshan S Prabhu ${ }^{3,4}$, Katherine Miller ${ }^{3}$ and Stuart Burriti,4

${ }^{1}$ Department of Neurosurgery, USA

${ }^{2}$ Carolina Neurosurgery and Spine Associates, USA

${ }^{3}$ Levine Cancer Institute, USA

${ }^{4}$ Southeast Radiation Oncology Group, USA

\section{Abstract}

Purpose: Management of high-grade gliomas currently relies heavily on a tissue diagnosis to guide treatment decisions, often obtained by stereotactic biopsy.Patients with unresectable high-grade gliomas have a dismal prognosis even with optimal therapy.Sparing them the cost, potential morbidity, and risk of biopsy may be in their best interest.

Method: All patients at one institution over a 9-year period (2005-2013) who had pathological confirmation of high-grade gliomas with stereotactic biopsy and did not undergo any additional surgery were included in this retrospective analysis.Data were collected on patient demographics, performance status (PS), overall survival (OS), 60-day mortality, and iatrogenic hemorrhage.

Result: 160 patients underwent analysis. Median OS was 5.1 months.46 patients died within 60 days after biopsy.8 patients suffered iatrogenic hemorrhage. Older age predicted worse OS and 60-day mortality. Combining PS and age provided superior prognostic information rather than age or PS alone.Patients with PS $0-2$ and age $\leq 65$ had a median OS of 10.1 months and $14 \%$ mortality within 60 days post-operatively.

Conclusion: High grade glioma patients with low performance status and/or advanced age may be better served by avoiding the cost and risk of stereotactic biopsy.Age has a strong effect on overall survival following biopsy in this cohort.In the setting of suspected unresectable high-grade glioma with poor performance status, advanced age, and low likelihood of improvement, there appears to be little gained from biopsy.Careful patient selection is necessary to avoid overtreatment in this population.

Keywords: Glioblastoma;Anaplastic astrocytoma;High-grade glioma;Stereotactic biopsy;Performance status; Inoperable brain tumor

\section{Introduction}

Management of high-grade gliomas currently relies heavily on a tissue diagnosis to guide treatment decisions Weller et al. [1]. For clinically and radiologically suspected high-grade gliomas that are not amenable to resection, stereotactic biopsy is the most common technique for obtaining a tissue sample. As with any operative procedure, if the risk of biopsy is too high, the results of are unlikely to substantively alter subsequent treatment, or the prognosis is likely to be unfavorable even with treatment, the potential morbidity and expense of biopsy should be avoided. However, the clinical guidelines for making the decision to biopsy or not remain poorly defined [2]. Empiric therapy is rarely initiated without a histological diagnosis. Overall, stereotactic biopsy has high diagnostic yield and accuracy with low risk [3-6]. However, these risks are not insignificant, and limited data has suggested that the location and histology of the tumor can influence the rate of operative morbidity. Deep seated location of the biopsy target is a clinically significant risk factor compared to superficial location, with morbidity ranging from $11 \%$ to $27 \%$ for deep lesions [7]. High-grade glioma is a significant risk factor 
for morbidity with stereotactic biopsy, with one study reporting $11 \%$ morbidity and an OR of 4.0 for high-grade gliomas compared to all other histologies [7]. Patients with deep seated, unresectable malignant lesions have a dismal prognosis even with optimal therapy, with mean survival as short as 6 months [8-11]. Sparing them the cost, potential morbidity, and risk of mortality from a stereotactic biopsy and anesthesia may be in their best interest. Careful patient selection is necessary to avoid overtreatment in this patient population. However, a consensus regarding the decision to pursue biopsy is not defined. The objective of this study is to define the utility of stereotactic biopsy more clearly in patients with unresectable high-grade gliomas by analyzing the morbidity and mortality of both the intervention and the natural progression of the disease. We hypothesize that there is a specific patient population that is overtreated with stereotactic biopsy alone and that no intervention with hospice consultation would be in their best interest.

\section{Method}

We retrospectively reviewed all patients at one institution over a 9-year period (2005-2013) who had pathological confirmation of high-grade gliomas (WHO grade III or IV) with stereotactic biopsy and did not undergo surgical resection or de-bulking of the lesion. The lesions were either not amenable to resection due to size and/ or eloquent location or the patient was a poor surgical candidate due to low performance status. Thus, stereotactic biopsy was performed rather than open biopsy and/or resection. IRB approval was obtained. Individual patient consent was not deemed necessary as it was a retrospective review of standard of care treatment and all identifying information was removed. The Eastern Cooperative Oncology Group (ECOG) scale of performance status (PS) was used to evaluate preoperative functional status in all patients (Table 1). Outcomes of interest were overall survival, death within 60 days post biopsy, and post-operative hemorrhage attributable to the biopsy. All patients had either a confirmed date of death or at least 5 years of follow up after the biopsy was performed. This information was primarily obtained through chart review of all clinical documentation. Phone calls were made to patients and/or their families for follow-up if there was not a confirmed date of death documented. Post-biopsy CT scans were reviewed retrospectively for signs of iatrogenic hemorrhage, although for some of the earliest patients in the series, these scans were not available for analysis. These endpoints were stratified by age and performance status (PS). PS was stratified by comparing patients with ECOG PS 3 or 4 to 0,1 , or 2 . Age was stratified in three ways, comparing 65 and younger to older than 65, 70 and younger to older than 70, and 75 and younger to older than 75 . The last stratification combined PS and age into four different groups: PS 3-4 and age greater than 65 , PS 3-4 and age less than or equal to 65, PS 0-2 and age greater than 65 , and PS 0-2 with age less than or equal to 65. Fisher's exact test was used for comparison between groups. Overall survival (OS) was estimated using the Kaplan-Meier method and log-rank tests were used to evaluate differences between groups.

Table 1: Eastern Cooperative Oncology Group (ECOG) scale of performance status (PS).

\begin{tabular}{|c|c|}
\hline ECOG & Description \\
\hline 0 & Fully active, able to carry on all pre-disease performance without restrictions \\
\hline 1 & Ambulatory and capable of all self-care but unable to carry out any work activities. Up and about more than 50\% of \\
\hline 2 & Capable of only limited self-care, confined to bed or chair more than 50\% of waking hours \\
\hline 3 & Completely disabled. Cannot carry on self-care. Totally confined to bed or chair. \\
\hline 4 &
\end{tabular}

\section{Result}

Patient characteristics: Initial chart review revealed 214 patients who underwent stereotactic biopsy for primary brain lesions. 160 patients were identified that met inclusion criteria. 51 were excluded for having a pathology other than WHO Grade III or IV glioma, and 3 patients were excluded due to subsequent surgical resection after stereotactic biopsy. At the time of analysis, 157 had a confirmed date of death, while 3 were still alive. All three patients that were still alive had at least 5 years of follow up after the biopsy. Of these 160 patients, there were 140 that had post-operative CT scans available for review to assess for iatrogenic hemorrhage. In addition, information on chemotherapy and radiotherapy was available for 128 of these patients. Table 2 provides a summary of patient demographics and baseline characteristics. The median age at biopsy was 64 years and the mean was 65 (range 18 to 91). Only five were under the age of 40 and only two were under the age of 30. The median OS for the entire cohort of patients was 5.1 months (95\% confidence interval $[\mathrm{CI}]=4.2-6.3$, Figure 1$)$. The median preoperative ECOG PS was 2, and 36 (22.5\%) patients had an ECOG PS of 3 or 4 .

Adverse events: The incidence of adverse events is summarized in Table 2. 46 (28.8\%) patients died within 60 days following biopsy. Eight $(5.7 \%)$ patients had iatrogenic hemorrhage confirmed on post-op CT scans. Two of these hemorrhages were not clinically significant and did not delay discharge, three were intra-cerebral hematomas that required additional imaging for observation in the hospital which delayed discharge, and three patients either died or went to hospice as a direct result of iatrogenic hemorrhage. 
Table 2: Patient demographics, characteristics, overall survival, adverse events.

\begin{tabular}{|c|c|c|}
\hline Characteristic & & $\mathrm{N}=160$ \\
\hline \multicolumn{3}{|c|}{ Age, years } \\
\hline Mean & & 65 \\
\hline Median & & 64 \\
\hline Range & & $18-91$ \\
\hline \multicolumn{3}{|c|}{ Age Group, n (\%) } \\
\hline$\leq 65$ years & & $86(53.7)$ \\
\hline$>65$ years & & $74(46.3)$ \\
\hline$<40$ years & & $5(3.1)$ \\
\hline \multicolumn{3}{|c|}{ ECOG PS, n (\%) } \\
\hline 0 & & $6(3.8)$ \\
\hline 1 & & $53(33.1)$ \\
\hline 2 & & $65(40.6)$ \\
\hline 3 & & $24(15.0)$ \\
\hline 4 & & $12(7.5)$ \\
\hline \multicolumn{3}{|c|}{ OS, months } \\
\hline Median $(95 \% \mathrm{CI})$ & & $5.1(4.2-6.3)$ \\
\hline \multicolumn{2}{|c|}{1 year OS rate } & $25.60 \%$ \\
\hline \multicolumn{3}{|c|}{ Lesion Type } \\
\hline WHO Grade IV Glioma & & 136 \\
\hline WHO Grade III Glioma & & 24 \\
\hline \multicolumn{3}{|c|}{ Adjuvant Therapy } \\
\hline Radiotherapy and Chemotherapy & & 84 \\
\hline Radiotherapy & & 18 \\
\hline No adjuvant therapy & & 26 \\
\hline Unknown & & 32 \\
\hline \multicolumn{3}{|c|}{ ECOG PS = Eastern Cooperative Oncology Group Performance Status } \\
\hline \multicolumn{3}{|l|}{ OS = Overall Survival } \\
\hline & Number of Events & Total Patients \\
\hline Death within 60 -days & 46 & 160 \\
\hline Iatrogenic bleeding event & 8 & 140 \\
\hline Clinically insignificant ICH & 2 & \\
\hline ICH that delayed discharge & 3 & \\
\hline Death or hospice from ICH & 3 & \\
\hline
\end{tabular}

Table 3: Effect of performance status and age on mortality.

\begin{tabular}{|c|c|c|c|c|c|c|c|c|c|c|}
\hline \multicolumn{6}{|c|}{ 60-Day Mortality } & \multirow{2}{*}{$\begin{array}{c}\text { Median OS } \\
\text { Months }\end{array}$} & \multirow{2}{*}{$\begin{array}{c}1 \text { Yr OS Rate } \\
\%\end{array}$} & \multirow[b]{2}{*}{ HR } & \multirow[b]{2}{*}{$95 \% \mathrm{CI}$} & \multirow[b]{2}{*}{ P-value } \\
\hline Variable & Subgroup & Total & \# & $\%$ & P-value & & & & & \\
\hline \multirow[t]{2}{*}{ ECOG PS } & $0-2$ & 124 & 32 & 25.8 & 0.145 & 5.4 & 25.8 & 1.2 & $0.82-1.75$ & 0.348 \\
\hline & $3-4$ & 36 & 14 & 38.9 & & 4 & 25 & & & \\
\hline \multirow[t]{4}{*}{ Age (yrs) } & $\leq 65$ & 86 & 18 & 20.9 & 0.023 & 9 & 39.5 & 2.26 & $1.62-3.16$ & 0.001 \\
\hline & $>65$ & 74 & 28 & 37.8 & & 3.7 & 9.5 & & & \\
\hline & $\leq 70$ & 106 & 26 & 24.5 & 0.139 & 7.9 & 34.9 & 2.3 & $1.62-3.26$ & 0.001 \\
\hline & $>70$ & 54 & 20 & 37 & & 3.2 & 7.4 & & & \\
\hline
\end{tabular}




\begin{tabular}{|c|c|c|c|c|c|c|c|c|c|c|}
\hline & $\leq 75$ & 121 & 29 & 24 & 0.025 & 7.5 & 32.2 & 2.57 & $1.74-3.79$ & 0.001 \\
\hline & $>75$ & 39 & 17 & 43.6 & & 2.6 & 5.1 & & & \\
\hline ECOG PS, Age (yrs) & $0-2 \leq 65$ & 64 & 9 & 14 & 0.006 & 10.1 & 42.2 & Ref & Ref & 0.001 \\
\hline & $0-2>65$ & 60 & 23 & 38.3 & & 3.4 & 8.3 & 2.39 & $1.65-3.47$ & \\
\hline & $3-4 \leq 65$ & 22 & 9 & 40.9 & & 4.2 & 31.8 & 1.37 & $0.83-2.25$ & \\
\hline & $3-4>5$ & 14 & 5 & 35.7 & & 4 & 14.3 & 2.57 & $1.41-4.66$ & \\
\hline
\end{tabular}

Stratified analyses: Table 3 shows the results of the subgroup analysis. PS was not significantly prognostic for death within 60 days or OS. Median OS for patients with ECOG PS 0-2 was 5.4 months and 3-4 was 4.0 months ( $\mathrm{p}=0.34)$. The risk of death within 60 days of biopsy for patients with an ECOG PS 3-4 was 38.9\% versus $25.8 \%$ for the $0-2$ group $(\mathrm{p}=0.145)$. Death within 60 days and OS was significantly worse for older patients (Table 3). For all three cutoff points $(65,70,75$ years) the older group had highly significant worse OS ( $p<0.001)$. The combination of age and performance status showed comparable poor outcomes for three of the four groups. PS 0-2 and age $>65$ had a $38.3 \% 60$-day mortality rate and median OS of 3.4 months. PS $3-4$ and age $\leq 65$ had a $40.9 \% 60$-day mortality rate and median OS of 4.2 months. PS 3-4 and age $>65$ had a $35.7 \%$ 60-day mortality rate and median os of 4.0 months. The fourth group had a much improved 60-day mortality rate and median overall survival that was highly significant compared to the other groups. PS $0-2$ and $\leq 65$ had a $14 \% 60$-day mortality rate $(\mathrm{p}=$ $0.006)$ and median OS of 10.1 months $(\mathrm{p}<0.001)$. Figure 1 shows Kaplan-Meier curves for OS. PS and age had no significant effect on risk of bleeding (data not shown).

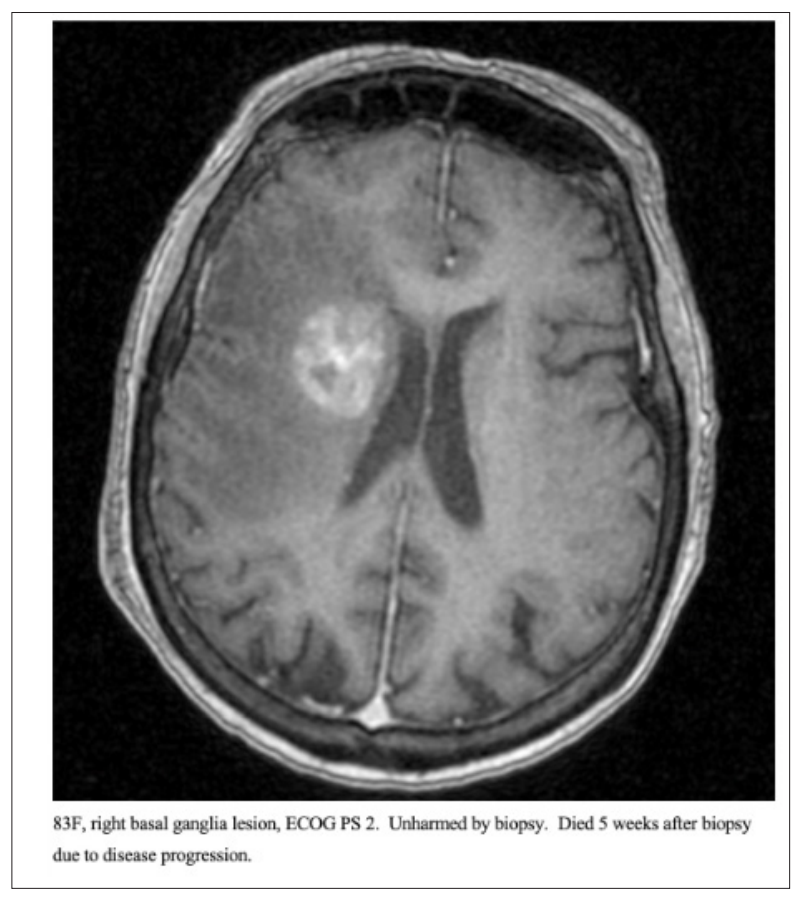

Figure 1: 83F, right basal ganglia lesion, ECOG PS 2. Unharmed by biopsy. Died 5 weeks after biopsy due to disease progression.

\section{Discussion}

This retrospective analysis characterized the iatrogenic hemorrhage rates for stereotactic biopsy and overall survival in a large series of patients at one institution. All of these patients had unresectable high-grade gliomas, and all were managed by experienced tumor surgeons. The term "unresectable" is debatable and beyond the scope of this paper. Figures 2-4 show imaging for several patients included in this cohort. Data on outcomes for unresectable high-grade gliomas following stereotactic biopsy is very limited. We identified variables associated with increased risk of poor postoperative outcomes. Age was a strong predictor of 60-day mortality and overall survival. PS discriminated for 60-day mortality and overall survival following biopsy, but this did not reach statistical significance. Neither PS nor age had any prognostic value for risk of bleeding given our few bleeding events. Performance status is a well-established prognostic factor in patients with glioma. One previous study showed that glioblastoma patients with preoperative Karnofsky Performance Scale (KPS) $\leq 50$ had a median OS of 1.8 months, while patients with KPS 60, 70,80 , and $\geq 90$ had a median OS of $2.8,4.8,7.3$, and 17.2 months, respectively $(\mathrm{HR}=1.24,95 \% \mathrm{CI}=1.06-1.44, \mathrm{P}=0.006)$. The total cohort in this study was 206 patients, 93 of which who underwent 
surgical resection and 113 who underwent biopsy. Our series did not demonstrate PS significantly affecting prognosis, likely due to the fact that all patients in our series had unresectable lesions, and OS was very poor for the entire population. Age had the strongest effect on the risk of death within 60 days and overall survival following biopsy. Older age predicted significantly higher likelihood of death within 60 days and worse OS. This is consistent with existing literature showing that increasing age is a negative prognostic factor for glioblastoma, although there is very limited data looking at outcomes only for unresectable high-grade gliomas. Our data shows that combining PS and age provides superior prognostic information rather than age or performance status alone. We identified one subgroup that significantly outlived the other three subgroups. PS $0-2$ and $\leq 65$ had a much-improved median OS compared to the other groups, while PS 0-2 and age > 65 , PS 3-4 and age $\leq 65$, and PS 3-4 and age $>65$ all had similarly very poor outcomes. The majority of studies describing the risks of stereotactic biopsy consider the morbidity and mortality directly attributable to the procedure itself. By tracking mortality within 60 days and OS, this study encapsulates patients who were harmed by the procedure and those who experienced clinical deterioration or death due to natural progression of their disease. These patients with an extremely poor prognosis likely represent a patient population for which stereotactic biopsy is at best, futile, and at worst, harmful. Our data suggests that if a patient is over 65 years old or has an ECOG PS of 3-4, their median OS is only 3 to 4 months, and these patients are likely better served with hospice care and/ or empiric radiation rather than a biopsy.

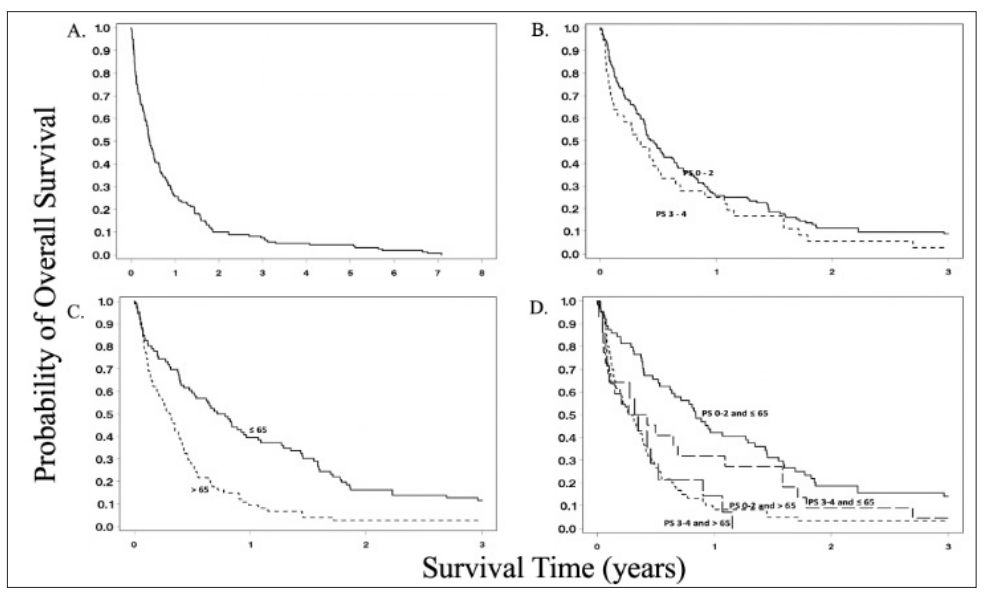

Figure 2: Kaplan-Meier curves for overall survival in the full cohort (A), stratified by PS (B), age (C), and age and PS (D).

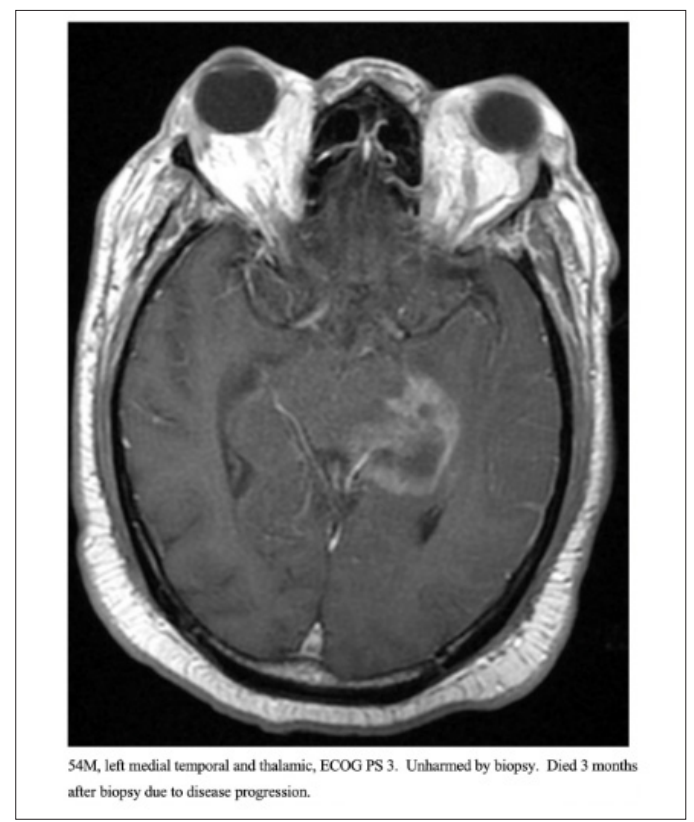

Figure 3: 54M, left medial temporal and thalamic, ECOG PS 3. Unharmed by biopsy. Died 3 months after biopsy due to disease progression. 


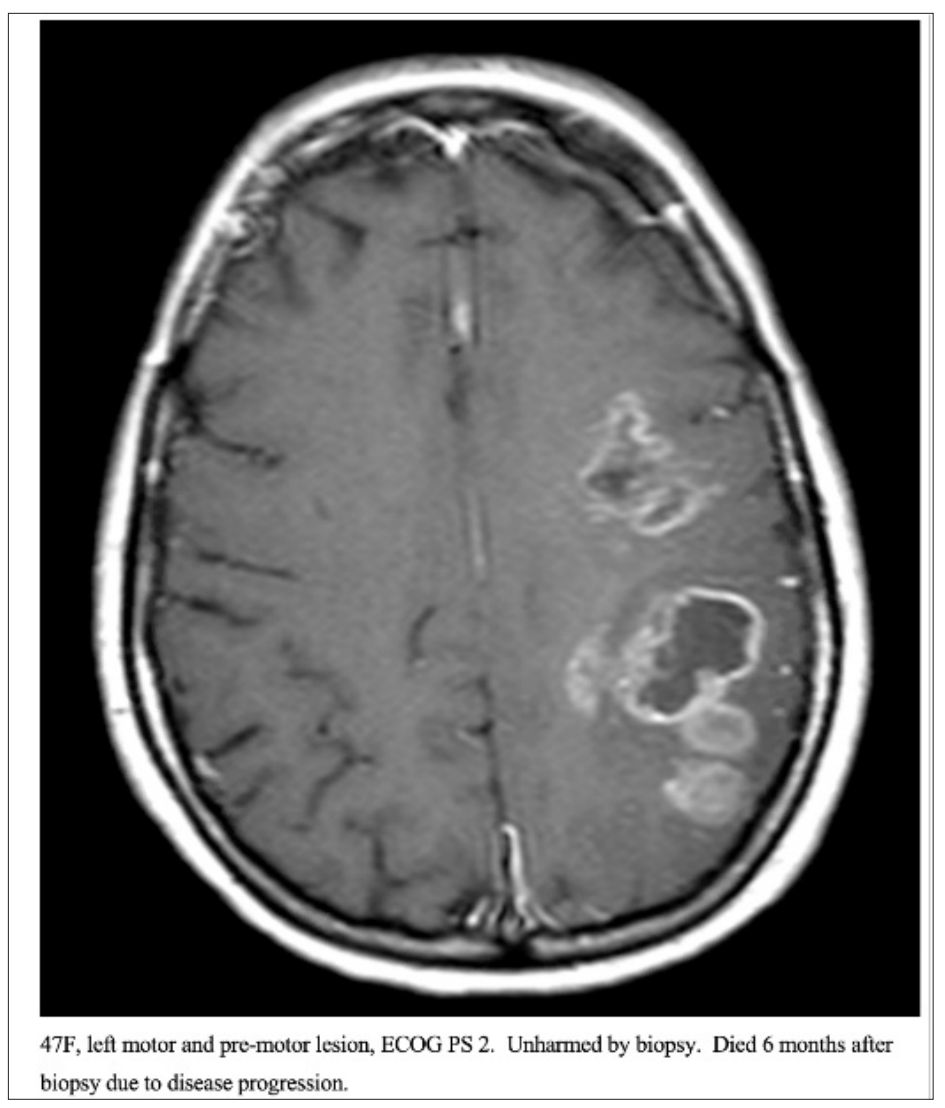

Figure 4: 47F, left motor and pre-motor lesion, ECOG PS 2. Unharmed by biopsy. Died 6 months after biopsy due to disease progression.

This study shows that for a specific patient population, there is a high rate of futility in performing stereotactic biopsy. All of the patients in this study had malignant gliomas confirmed by biopsy. For patients with clinically suspected malignant gliomas that are not yet histologically confirmed, the primary risk of foregoing biopsy is missing the diagnosis of a more treatable disease, such as lymphoma, which can be mistaken for high-grade glioma. However, patients with lymphoma often show significant benefit with the addition of steroids, which may improve their performance and alter the management approach suggested. Additionally, empiric radiation therapy is a reasonable therapeutic option for patients with CNS lymphoma and poor performance status. Further studies to characterize the accuracy of non-invasive methods (such as advanced imaging techniques) in predicting the diagnosis and/ or prognosis for suspected malignant gliomas without biopsy confirmation would be very helpful determining whether a patient should get a stereotactic biopsy of a deep seated, multi-focal or otherwise unresectable lesion already suspected to be malignant glioma, or instead proceed with hospice care and/or empiric radiation. Additionally, further work characterizing prognosis of high-grade, unresectable gliomas based on histological markers such MGMT would be helpful in the decision-making process. Variation between surgeons in what defines an "unresectable" lesion is likely the most important factor to consider regarding the generalizability of our results. Recently, the preponderance of (largely retrospective) data related to surgical therapies for gliomas has supported more aggressive (i.e., complete) surgical resections of these tumors when such resections can be accomplished without significant compromise of baseline neurological function [12-14]. As such we, like most other high-volume brain tumor centers, have steadily adopted a more aggressive stance for offering resection of glial neoplasms. In that regard, the population of glioma patients who undergo biopsy only in this institution (and likely many others) represents a progressively smaller cohort possessing less favorable lesions (e.g., multi-focal, deep, eloquent) and/or less favorable performance status-therefore, a less favorable prognosis. Given this general condition, and in consideration of the data presented here, we believe that a choice to proceed with biopsy only, particularly in older patients with poor performance status, should be met with a very high threshold.

\section{Conclusion}

High-grade glioma patients with low performance status and/or advanced age may be better served by avoiding the cost and risk of stereotactic biopsy. The primary advantage of tissue confirmation is to assess the role of chemotherapy and to guide more definitive therapy. Patients with poor performance are generally not candidates for chemotherapy and were excluded from 
the trials showing benefit Stupp [15]. In the setting of suspected unresectable high-grade glioma with poor performance status, advanced age, and low likelihood of improvement, there appears to be little gained from biopsy, and a real risk of harm. Given the extremely poor prognosis in this cohort, short-course palliative empiric radiotherapy or hospice referral should be carefully considered.

\section{Ethics Approval}

IRB approval was obtained.

\section{Patient Consent}

Individual patient consent was not deemed necessary as it was a retrospective review of standard of care treatment and all identifying information was removed. Availability of data: The datasets generated during and analyzed during the current study are available from the corresponding author on reasonable request.

All authors contributed to the study conception and design. Material preparation, data collection and analysis were performed by David Peters, James Symanowski, and Katherine Miller. The first draft of the manuscript was written by David Peters and all authors commented on previous versions of the manuscript. All authors read and approved the final manuscript.

\section{References}

1. Weller, Michael, Martin V, Jörg CT, Roger Stupp, et al. (2017) European association for neuro-oncology (EANO) guideline on the diagnosis and treatment of adult astrocytic and oligodendroglial gliomas. Lancet Oncol 18(6): e315-e329.

2. Mitchell, Patrick, David WE, David M (2005) Surgery for malignant gliomas: mechanistic reasoning and slippery statistics. Neurology 4(7): 413-422.

3. Chen, Ching, Peng WH, Tai WEW, Shih TL, et al. (2009) Stereotactic brain biopsy: Single center retrospective analysis of complications. Clinical Neurology and Neurosurgery 111(10): 835-839.
4. Dammers R, Haitsma K, Schouten JW, Kros JW, Avezaat CJJ, et al. (2008) Safety and efficacy of frameless and frame-based intracranial biopsy techniques. Acta Neurochir 150(1): 23-29.

5. Kellermann, Stephanie G, Christina AH, Daniel Rueß, Tobias Blau, et al. (2017) Stereotactic biopsy in elderly patients: risk assessment and impact on treatment decision. J Neuroncol 134(2): 303-307.

6. McGirt, Matthew J, Graeme FW, Alex LC, James MF, et al. (2005) Independent predictors of morbidity after image-guided stereotactic brain biopsy: a risk assessment of 270 cases. J Neurosurg 102(5): 897901.

7. Sawin P (1998) Computed imaging-assisted stereotactic brain biopsy a risk analysis of 225 consecutive cases. Surg Neurol 49(6): 640-649.

8. Rahman, Maryam, Joseph A, Edward K, Paul SK, et al. (2016) The effects of new or worsened postoperative neurological deficits on survival of patients with glioblastoma. J Neurosurg 127(1): 123-131.

9. Scott JG, Suh P, Barnett MA, Vogelbaum DM, Peereboom GHJ, et al. (2011) Aggressive treatment is appropriate for glioblastoma multiforme patients 70 years old or older: a retrospective review of 206 cases. Neuro Oncol 13(4): 428-436.

10. Stark, Andreas M, Julia V, Jürgen H, Maximilian M, et al. (2012) Glioblastoma: clinical characteristics, prognostic factors and survival in 492 patients. Clin Neurol Neurosurg 114(7): 840-845.

11. Filippini GC, Falcone, Boiardi, Broggi, Bruzzone, et al. (2008) Prognostic factors for survival in 676 consecutive patients with newly diagnosed primary glioblastoma. Neuro Oncol 10(1): 79-87.

12. Brown, Timothy J, Matthew CB, Michael L, Ephraim WC, et al. (2016) Association of the extent of resection with survival in glioblastoma. JAMA Oncol 2(11): 1460-1469.

13. Grabowski, Matthew M, Pablo FR, Amy SN, Jason L, et al. (2014) Residual tumor volume versus extent of resection: predictors of survival after surgery for glioblastoma. JNS 121: 1115-1123.

14. Li, Michael Y, Suki D, Hess K, Sawaya R (2016) The influence of maximum safe resection of glioblastoma on survival in 1229 patients: Can we do better than gross-total resection? J Neurosurg 124(4): 977-988.

15. Stupp, Roger, Monika EH, Warren PM, Martin J, et al. (2009) Effects of radiotherapy with concomitant and adjuvant temozolomide versus radiotherapy alone on survival in glioblastoma in a randomised phase III study: 5-year analysis of the EORTC-NCIC trial. Lancet Oncol 10: 459466.

For possible submissions Click below: 